\title{
APPLICATION OF A Z-TRANSFORMS METHOD FOR INVESTIGATION OF MARKOV G-NETWORKS
}

\author{
Mikhail Matalytski ${ }^{1}$, Victor Naumenko ${ }^{2}$ \\ ${ }^{I}$ Institute of Mathematics, Czestochowa University of Technology \\ Czestochowa, Poland \\ ${ }^{2}$ Faculty of Mathematics and Computer Science, Grodno State University \\ Grodno, Belarus \\ Im.matalytski@gmail.com,2victornn86@gmail.com
}

\begin{abstract}
The purpose of this research paper is to find the expected incomes in open Markov queueing networks with incomes, positive and negative messages at any time by the multidimensional transformations. Investigations were carried out in cases when incomes from the network transitions between the states are deterministic functions not dependent on network states and time. It is assumed that all network systems are one-line. It was proved the theorem on the expression for the multidimensional $z$-transform. An algorithm was proposed for calculation of expected incomes. It is calculated an example on the PC.
\end{abstract}

Keywords: open G-network, positive and negative messages, expected incomes, z-transforms method

\section{Introduction}

Markov networks with incomes (HM-network) and G-networks are widely used as mathematical models of various objects in computer sciences and other fields [1-3]. For example, G-network can be used to model the behavior of viruses in the information and telecommunication systems and networks for forecasting expenditure when it is in contact with viruses $[4,5]$. In this case an important task is to find such expenditures in the systems of the considered network. Previously in work [6] for finding the expected incomes of systems of HM-network with many-type messages networks a method was proposed of successive approximations, combined with the method of series. In it was considered a closed exponential HM-network with limited waiting times in queues queueing messages. In [7], it is proposed a method of finding the expected incomes of systems of HM-open queueing network (QN) with a one-line systems of QN (QS), positive and negative messages in the case, when the incomes from the state transitions are deterministic network functions depending on network states does not depend on time. Such a technique to find the expected network incomes of systems via PC for an infinite number of network states in a reasonable CPU time. 
In this paper, we consider another method based on the application of multivariate $z$-transforms. If we introduce into consideration multidimensional $z$-transforms for expected network incomes of systems, we obtain for their relations in this way, as in $[1,8]$. On the basis of these relations we can propose an algorithm for calculating the expected incomes $[9,10]$ for the open G-network with incomes.

\section{QN description. Formulation of the problem}

Consider an open QN G-network with $n$ one-line QS. In QN $S_{i}$ come from outside independent Poisson flows of positive and negative messages with intensities $\lambda_{0 i}^{+}$and $\lambda_{0 i}^{-}$respectively, $i=\overline{1, n}$. The service time of positive messages in the QS $S_{i}$ is distributed exponentially with parameter $\mu_{i}, i=\overline{1, n}$. The actions of negative messages are described in [2-5]. Positive message serviced in the QS $S_{i}$, with probability $p_{i j}^{+}$is directed to QS $S_{j}$ as positive message, and with probability $p_{i j}^{-}$- as negative message, and with probability $p_{i 0}=1-\sum_{j=1}^{n}\left(p_{i j}^{+}+p_{i j}^{-}\right)$message leaves the network to the external environment (QS $\left.S_{0}\right), i, j=\overline{1, n}$. The message during the transition from one to another QS brings its system, and some income of the first system is reduced by this amount respectively. Consider the case when the income from the state transitions are deterministic functions of the network, depending on network states. The network state will be the vector $k(t)=(k, t)=\left(k_{1}(t), k_{2}(t), \ldots, k_{n}(t)\right)$, where $k_{i}(t)$ - the number of messages at the time moment $t$ in system $S_{i}, i=\overline{1, n}$.

Let $v_{i}(k, t)$ - the total expected income, which gets the system $S_{i}$ during time $t$, if at the initial moment the network is in a state $k$, and assume that this function is differentiable in $t$. We introduce the following notations: $I_{i}$ - a vector of dimension $n$, consisting of zeros, except for the component with number of $i$, which is equal to $1, i=\overline{1, n} ; \quad I_{0}$ - zero $n$-vector; $u(x)=\left\{\begin{array}{l}1, x>0 \\ 0, x \leq 0\end{array}\right.$ - Heaviside function; $r_{i}(k)$ income system $S_{i}$ at a time when the network is in a state $k$. This means that, for example, if the network is in a state $k$ at moment time $t+\Delta t$, then the expected income of system $S_{i}$ will be $r_{i}(k) \Delta t$ during $\Delta t$ plus the expected income $v_{i}(k, t)$, that the system has received in the previous $t$ time units. Let $r_{0 i}\left(k+I_{i}\right)$ - income of system $S_{i}$, when network makes a transition from state $(k, t)$ in state $\left(k+I_{i}, t+\Delta t\right)$ during $\Delta t ;-R_{i 0}\left(k-I_{i}\right)$ - income of its system, if network makes a transition from state $(k, t)$ in state $\left(k-I_{i}, t+\Delta t\right) ; r_{i j}\left(k+I_{i}-I_{j}\right)$ - the income 
of system $S_{i}$, when the network makes its transition from state $(k, t)$ to $\left(k+I_{i}-I_{j}, t+\Delta t\right)$ during $\Delta t ;-r_{i j}\left(k-I_{i}-I_{j}\right)$ - income of QS $S_{i}$, when the network changes transition from state $(k, t)$ to $\left(k-I_{i}-I_{j}, t+\Delta t\right)$ during time $\Delta t$, $i, j=\overline{1, n}$.

It has been shown that the system of differential equations for the income $v_{i}(k, t)$ has the form $[4,5]$ :

$$
\begin{gathered}
\frac{d v_{i}(k, t)}{d t}=\alpha_{i}(k)-\sum_{j=1}^{n}\left[\lambda_{0 j}^{+}+\left(\lambda_{0 j}^{-}+\mu_{j}\right) u\left(k_{j}\right)\right] v_{i}(k, t)+ \\
+\sum_{j=1}^{n}\left(\lambda_{0 j}^{+} v_{i}\left(k+I_{j}, t\right)+\left[\mu_{j} p_{j 0} u\left(k_{j}\right)+\lambda_{0 j}^{-} u\left(k_{j}\right)+\mu_{j} \sum_{\substack{c=1 \\
c \neq j}}^{n} p_{j c}^{-}\left(1-u\left(k_{c}\right)\right)\right] v_{i}\left(k-I_{j}, t\right)\right]+ \\
+\lambda_{0 i}^{+} v_{i}\left(k+I_{i}, t\right)+\left[\mu_{i} p_{i 0} u\left(k_{i}\right)+\lambda_{0 i}^{-} u\left(k_{i}\right)+\mu_{i} \sum_{\substack{c=1 \\
c \neq i}}^{n} p_{i c}^{-}\left(1-u\left(k_{c}\right)\right)\right] v_{i}\left(k-I_{i}, t\right)+ \\
+\sum_{\substack{j=1 \\
j \neq i}}^{n}\left[\mu_{j} p_{j i}^{+} u\left(k_{j}\right) v_{i}\left(k+I_{i}-I_{j}, t\right)+\mu_{i} p_{i j}^{+} u\left(k_{i}\right) v_{i}\left(k-I_{i}+I_{j}, t\right)+\right. \\
\left.+\mu_{i} p_{i j}^{-} v_{i}\left(k-I_{i}-I_{j}, t\right)\right]+\sum_{\substack{c, s=1 \\
c, s \neq i, c \neq s}}^{n}\left[\mu_{s} p_{s c}^{+} u\left(k_{s}\right) v_{i}\left(k+I_{c}-I_{s}, t\right)+\mu_{c} p_{c s}^{-} v_{i}\left(k-I_{c}-I_{s}, t\right)\right],
\end{gathered}
$$

where

$$
\begin{gathered}
\alpha_{i}(k)=r_{i}(k)+\lambda_{0 i}^{+} r_{0 i}\left(k+I_{i}\right)- \\
-\left[\mu_{i} p_{i 0} u\left(k_{i}\right)+\lambda_{0 i}^{-} u\left(k_{i}\right)+\mu_{i} \sum_{\substack{c=1 \\
c \neq i}}^{n} p_{i c}^{-}\left(1-u\left(k_{c}\right)\right)\right] R_{i 0}\left(k-I_{i}\right)+ \\
+\sum_{\substack{j=1 \\
j \neq i}}^{n}\left[\mu_{j} p_{j i}^{+} u\left(k_{j}\right) r_{j i}\left(k+I_{i}-I_{j}\right)-\mu_{i} p_{i j}^{+} u\left(k_{i}\right) r_{i j}\left(k-I_{i}+I_{j}\right)-\right. \\
\left.-\mu_{i} p_{i j}^{-} r_{i j}\left(k-I_{i}-I_{j}\right)\right], i=\overline{1, n} .
\end{gathered}
$$

It is necessary to find the expected network incomes of systems during $t$, if we know its state at the initial time. 


\section{Analysis of incomes in network by $z$-transforms method}

We introduce multidimensional $z$-tranforms for expected income of system $S_{i}$ :

$$
\varphi_{i}(z, t)=\sum_{\substack{k_{i}=0 \\ i=1, n}}^{\infty} v_{i}(k, t) \prod_{l=1}^{n} z_{l}^{k_{l}}, z \in\left\{\left(z_{1}, z_{2}, \ldots, z_{n}\right) /\left|z_{i}\right|<1, i=\overline{1, n}\right\} .
$$

Multiply the system of equations (2) to $\prod_{l=1}^{n} z_{l}^{k_{l}}$ and summing over all $k_{l}$, $l=\overline{1, n}$, from 0 to $\infty$. Consider some sums included into converted system of equations. It can be shown that

$$
\begin{gathered}
\sum_{1 j}(z, t)=\sum_{\substack{k_{l}=0, l=1, n}}^{\infty} \sum_{j=1}^{n}\left[\lambda_{0 j}^{+}+\left(\lambda_{0 j}^{-}+\mu_{j}\right) u\left(k_{j}\right)\right] v_{i}(k, t) z_{1}^{k_{1}} z_{2}^{k_{2}} \cdot \ldots \cdot z_{n}^{k_{n}}= \\
=\sum_{j=1}^{n}\left[\lambda_{0 j}^{+}+\lambda_{0 j}^{-}+\mu_{j}\right]\left(\varphi_{i}(z, t)-\varphi_{i}^{\{j\}}(z, t)\right), j=\overline{1, n},
\end{gathered}
$$

where

$$
\begin{gathered}
\varphi_{i}^{\{j\}}(z, t)=\sum_{k_{1}=0}^{\infty} \ldots \sum_{k_{j-1}=0}^{\infty} \sum_{k_{j+1}=0}^{\infty} \ldots \sum_{k_{n}=0}^{\infty} v_{i}\left(k_{1}, \ldots, k_{j-1}, 0, k_{j+1}, \ldots k_{n}, t\right) z_{1}^{k_{1}} \cdot \ldots \cdot z_{j-1}^{k_{j-1}} z_{j+1}^{k_{j+1}} \cdot \ldots \cdot z_{n}^{k_{n}}= \\
=\left.\sum_{\substack{k_{l}=0, l=1, n, l \neq j}}^{\infty} v_{i}(k, t)\right|_{k_{j}=0} \prod_{\substack{l=1, l \neq j}}^{n} z_{l}^{k_{l}}, j=\overline{1, n} . \\
\sum_{2 j}(z, t)=\sum_{\substack{k_{l}=0, j=1 \\
l=1, n}}^{\infty} \sum_{j}^{n}\left[\mu_{j} p_{j 0} u\left(k_{j}\right)+\lambda_{0 j}^{-} u\left(k_{j}\right)+\mu_{j} \sum_{\substack{c=1 \\
c \neq j}}^{n} p_{j c}^{-}\left(1-u\left(k_{c}\right)\right)\right] v_{i}\left(k-I_{j}, t\right) \prod_{l=1}^{n} z_{l}^{k_{l}}= \\
=\sum_{j=1}^{n}\left[\mu_{j} p_{j 0}+\lambda_{0 j}^{-}\right] z_{j} \varphi_{i}(z, t), j=\overline{1, n} . \\
\sum_{3 j}(z, t)=\sum_{\substack{k_{l}=0,0 \\
l=1, n}}^{\infty} \sum_{j=1}^{n} \lambda_{0 j}^{+} v_{i}\left(k+I_{j}, t\right) \prod_{l=1}^{n} z_{l}^{k_{l}}=\sum_{j=1}^{n} \frac{\lambda_{0 j}^{+}}{z_{j}}\left[\varphi_{i}(z, t)-\varphi_{i}^{\{j\}}(z, t)\right], i=\overline{1, n} . \\
\sum_{4 j}(z, t)=\sum_{\substack{k_{l}=0, j=1 \\
l=1, n}}^{\infty} \sum_{j \neq i}^{n}\left[\mu_{j} p_{j i}^{+} u\left(k_{j}\right) v_{i}\left(k+I_{i}-I_{j}, t\right)\right] v_{i}\left(k+I_{i}-I_{j}, t\right) \prod_{l=1}^{n} z_{l}^{k_{l}}= \\
=\sum_{\substack{j=1 \\
j \neq i}}^{n} \mu_{j} p_{j i}^{+} \frac{z_{j}}{z_{i}}\left[\varphi_{i}(z, t)-\varphi_{i}^{\{i\}}(z, t)\right], i=\overline{1, n} .
\end{gathered}
$$




$$
\begin{aligned}
& \sum_{5 j}(z, t)=\sum_{\substack{k_{l}=0, j=1 \\
l=1, n}}^{\infty} \sum_{j \neq i}^{n} \mu_{i} p_{i j}^{+} u\left(k_{i}\right) v_{i}\left(k+I_{j}-I_{i}, t\right) \prod_{l=1}^{n} z_{l}^{k_{l}}= \\
& =\sum_{\substack{j=1 \\
j \neq i}}^{n} \mu_{i} p_{i j}^{+} \frac{z_{i}}{z_{j}}\left[\varphi_{i}(z, t)-\varphi_{i}^{\{j\}}(z, t)\right] i, j=\overline{1, n} . \\
& \sum_{6 s}(z, t)=\sum_{\substack{k_{l}=0, l=1, n}}^{\infty} \sum_{\substack{c, s=1 \\
l \neq i, c \neq s}}^{n} \mu_{s} p_{s c}^{+} u\left(k_{s}\right) v_{s}\left(k+I_{c}-I_{s}, t\right) \prod_{l=1}^{n} z_{l}^{k_{l}}= \\
& =\sum_{\substack{c, s=1 \\
c, s \neq i, c \neq s}}^{n} \mu_{s} p_{s c}^{+} \frac{z_{s}}{z_{c}}\left[\varphi_{c}(z, t)-\varphi_{c}^{\{c\}}(z, t)\right], c, s=\overline{1, n}, c, s \neq i . \\
& \sum_{7 j}(z, t)=\sum_{\substack{k_{l}=0 \\
l=1, n}}^{\infty} \sum_{\substack{j=1 \\
j \neq i}}^{n} \mu_{i} p_{i j}^{-} v_{i}\left(k-I_{i}-I_{j}, t\right) \prod_{l=1}^{n} z_{l}^{k_{l}}= \\
& =\sum_{\substack{j=1 \\
j \neq i}}^{n} \mu_{i} p_{i j}^{-}\left[\varphi_{i}(z, t)-\varphi_{i}^{\{i\}}(z, t)-\varphi_{i}^{\{j\}}(z, t)\right], j=\overline{1, n} . \\
& \sum_{8 j}(z, t)=\sum_{\substack{k_{l}=0, l=1, n}}^{\infty} \sum_{\substack{c, s=1 \\
c, s \neq i, c \neq s}}^{n} \mu_{s} p_{s c}^{+} v_{i}\left(k-I_{c}-I_{s}, t\right) \prod_{l=1}^{n} z_{l}^{k_{l}}= \\
& =\sum_{\substack{c, s=1 \\
c, s \neq i, c \neq s}}^{n} \mu_{s} p_{s c}^{+}\left[\varphi_{c}(z, t)-\varphi_{c}^{\{c\}}(z, t)-\varphi_{c}^{\{s\}}(z, t)\right], c, s=\overline{1, n}, c, s \neq i .
\end{aligned}
$$

Thus, using (3)-(10), the system of equations (1) after the above transformations we got approval.

Theorem. The function $\varphi_{i}(z, t)$ satisfies the relation

$$
\begin{aligned}
& \frac{\partial \varphi_{i}(z, t)}{\partial t}=\sum_{j=1}^{n}\left\{-\left(\lambda_{0 j}^{+}+\lambda_{0 j}^{-}+\mu_{j}\right)\left[\varphi_{i}(z, t)-\varphi_{i}^{\{j\}}(z, t)\right]+\frac{\lambda_{0 j}^{+}}{z_{j}}\left[\varphi_{i}(z, t)-\varphi_{i}^{\{j\}}(z, t)\right]+\right. \\
&\left.+\left(\mu_{j} p_{j 0}+\lambda_{0 j}^{-}\right) z_{j} \varphi_{i}(z, t)\right\}+\frac{\lambda_{0 i}^{+}}{z_{i}}\left[\varphi_{i}(z, t)-\varphi_{i}^{\{i\}}(z, t)\right]+\left(\mu_{i} p_{i 0}+\lambda_{0 i}^{-}\right) z_{i} \varphi_{i}(z, t)+ \\
&+\sum_{\substack{j=1 \\
j \neq i}}^{n}\left\{\frac{\mu_{j} p_{j i}^{+} z_{j}}{z_{i}}\left[\varphi_{i}(z, t)-\varphi_{i}^{\{i\}}(z, t)\right]+\frac{\mu_{i} p_{i j}^{+} z_{i}}{z_{j}}\left[\varphi_{i}(z, t)-\varphi_{i}^{\{j\}}(z, t)\right]+\right.
\end{aligned}
$$




$$
\begin{gathered}
\left.+\mu_{i} p_{i j}^{-}\left[\varphi_{i}(z, t)-\varphi_{i}^{\{i\}}(z, t)-\varphi_{i}^{\{j\}}(z, t)\right]\right\}+\sum_{\substack{c, s=1, c, s \neq i}}^{n}\left\{\frac{\mu_{s} p_{s c}^{+} z_{s}}{z_{c}}\left[\varphi_{c}(z, t)-\varphi_{c}^{\{c\}}(z, t)\right]+\right. \\
\left.+\mu_{c} p_{c s}^{-}\left[\varphi_{i}(z, t)-\varphi_{i}^{\{c\}}(z, t)-\varphi_{i}^{\{s\}}(z, t)\right]\right\}+\sum_{\substack{k_{l}=0 \\
l=1, n}}^{\infty} \alpha_{i}(k) \prod_{l=1}^{n} z_{l}^{k_{l}} .
\end{gathered}
$$

Consider the last sum in (12). Suppose that incomes $r_{i}(k), r_{i j}(k), r_{0 i}(k)$, $R_{i 0}(k)$ do not depend on network states $k$, i.e. $r_{i}(k)=r_{i}, r_{i j}(k)=r_{i j}, r_{0 i}(k)=r_{0 i}$, $R_{i 0}(k)=R_{i 0}$. Since the $\sum_{\substack{k_{l}=0, l=1, n}}^{\infty} \prod_{l=1}^{n} z_{l}^{k_{l}}=\prod_{l=1}^{n} \frac{1}{1-z_{l}}$,

in this case

$$
\sum_{\substack{k_{l}=0 \\ l=1, n}}^{\infty} \alpha_{i}(k) \prod_{l=1}^{n} z_{l}^{k_{l}}=\left\{r_{i}+\lambda_{0 i}^{+} r_{0 i}-\left(\mu_{i} p_{i 0}+\lambda_{0 i}^{-}\right) R_{i 0}+\sum_{\substack{j=1 \\ j \neq i}}^{n}\left[\mu_{j} p_{j i}^{+} r_{j i}-\mu_{i} p_{i j}^{+} r_{i j}-\mu_{i} p_{i j}^{-} r_{i j}\right]\right\} \prod_{l=1}^{n} \frac{1}{1-z_{l}} .
$$

Let $i_{1} \neq i_{2} \neq \ldots \neq i_{j}$. We introduce some notation related to $z$-transforms of incomes of systems $S_{i}$ :

$$
\begin{aligned}
& \varphi_{i}^{\Omega}(z, t)=\left.\varphi_{i}(z, t)\right|_{k_{j}=0, z_{j}=1, j=\overline{1, n}}=v_{i}(0,0, \ldots, 0, t) ; \\
& \varphi_{i}^{\Omega \backslash\left\{i_{i}\right\}}(z, t)=\left.\varphi_{i}(z, t)\right|_{k_{j}=0, z_{j}=1, j=\overline{1, n,}, j \neq i_{1}}=\sum_{k_{i_{1}}=0}^{\infty} v_{j}\left(0, \ldots, 0, k_{i_{1}}, 0, \ldots, 0, t\right) z_{i_{1}}^{k_{i_{1}}}, i_{1}=\overline{1, n} ; \\
& \varphi_{i}^{\Omega \backslash\left\{i_{1}, i_{2}\right\}}(z, t)=\left.\varphi_{i}(z, t)\right|_{k_{j}=0, z_{j}=1, j=1, n, j \neq i_{1}, i_{2}}=\sum_{k_{i_{1}}=0}^{\infty} \sum_{i_{i_{2}}=0}^{\infty} v_{i}\left(0, \ldots, 0, k_{i_{1}}, 0, \ldots, 0, k_{i_{2}}, 0, \ldots, 0, t\right) z_{i_{1}}^{k_{i_{1}}} z_{i_{2}}^{k i_{2}}, \\
& i_{1}, i_{2}=\overline{1, n}, i_{1} \neq i_{2} \\
& \varphi_{i}^{\Omega \backslash\left\{i_{1}, i_{2}, \ldots, i_{l}\right\}}(z, t)=\left.\dot{\varphi}_{i}(z, t)\right|_{k_{j}=0, z_{j}=1, j=1, \bar{n}_{, j \neq i_{1}, i_{2}, \ldots, i_{l}}}= \\
& =\sum_{k_{i_{1}}=0 k_{i_{2}}=0}^{\infty} \sum_{k_{i_{l}}=0}^{\infty} v_{i}^{\infty}\left(0, \ldots, 0, k_{i_{1}}, 0, \ldots, 0, k_{i_{2}}, 0, \ldots, 0, k_{i_{l}}, 0, \ldots, 0, t\right) z_{i_{1}}^{k_{i_{1}}} z_{i_{2}}^{k_{i_{2}}} \cdot \ldots \cdot z_{i_{j}}^{k_{i l}}, \\
& i_{1}, i_{2}, \ldots, i_{l}=\overline{1, n}, i_{1} \neq i_{2} \neq \ldots \neq i_{l} \\
& \varphi_{i}^{\Omega \backslash\left\{i_{1}, i_{2}, \ldots, i_{n-1}\right\}}(z, t)=\left.\varphi_{i}(z, t)\right|_{k_{j}=0, z_{j}=1, j=\overline{1, n}, j \neq i_{1}, i_{2}, \ldots, i_{n-1}}=\varphi_{i}^{\{j\}}(z, t), i=\overline{1, n}, \\
& i_{1} \neq i_{2} \neq \ldots \neq i_{n-1} .
\end{aligned}
$$


And in the function arguments $v_{i}$ between $k_{i_{j}}$ zeros can not stand, it is only important to $i_{1} \neq i_{2} \neq \ldots \neq i_{j}$. We present an algorithm for finding the expected incomes, using the relation (12).

\section{Algorithm for finding of incomes}

0-Step: it is necessary to define $\varphi_{i}^{\Omega}(z, t)=v_{i}(0,0, \ldots, 0, t)$.

1st-Step: find $z$-transforms $\varphi_{i}^{\Omega\left\{\left\{i_{1}\right\}\right.}(z, t)$, i.e. $z$-transformation of income of system $S_{i}$ at moment time $t$, at the initial time it is located in a state $k=\left(0,0, \ldots, k_{i_{1}}, 0, \ldots, 0\right)$. To do this, multiply the equation (3) on $\prod_{l=1}^{n} z_{l}^{k_{l}}$ and summing over all $k_{l}, l=\overline{1, n}$, from 0 to $\infty$. Taking into account that $k_{l}=0, l \neq i_{1}$ the sums of (3)-(10) can be rewritten in the following form:

$$
\begin{gathered}
\sum_{\substack{k_{l}=0, l=1, n}}^{\infty} v_{i}(k, t) z_{1}^{k_{1}} z_{2}^{k_{2}} \cdot \ldots \cdot z_{n}^{k_{n}}=\left\{\begin{array}{l}
0, j \neq i_{1}, \\
\varphi_{i}^{\Omega \backslash\left\{i_{1}\right\}}(z, t), j=i_{1},
\end{array}, j=\overline{1, n} .\right. \\
\sum_{\substack{k_{l}=0, l=1, n}}^{\infty} v_{i}\left(k-I_{j}, t\right) \prod_{l=1}^{n} z_{l}^{k_{l}}=\sum_{\substack{k_{l}=0, l=1, n}}^{\infty} v_{i}(k, t) z_{j} \prod_{l=1}^{n} z_{l}^{k_{l}}=\left\{\begin{array}{l}
0, j \neq i_{1}, \\
z_{i_{1}} \varphi_{j}^{\Omega \backslash\left\{i_{1}\right\}}(z, t), j=i_{1},
\end{array}, j=\overline{1, n} .\right. \\
\sum_{\substack{k_{l}=0, l=1, n}}^{\infty} v_{i}\left(k+I_{j}, t\right) \prod_{l=1}^{n} z_{l}^{k_{l}}=\left\{\begin{array}{c}
0, \\
\frac{1}{z_{i_{1}}}\left(\varphi_{i}^{\Omega \backslash\left\{i_{1}\right\}}(z, t)-v_{i}(0,0, \ldots, 0, t)\right), \\
j=i_{1},
\end{array}, j=\overline{1, n} .\right.
\end{gathered}
$$

Obviously, it is equal to zero if $j \neq i_{1}$, because $k_{j}=0$ at $j \neq i_{1}$. Consider this sum for $j=i_{1}$

$$
\sum_{4 i_{1}}(z, t)=\sum_{\substack{k_{l}=0 \\ l=1, n}}^{\infty} v_{i}\left(k+I_{i}, t\right) \prod_{l=1}^{n} z_{l}^{k_{l}} z_{i_{1}}=z_{i_{1}} \sum_{3 i}(z, t)=0 .
$$

If $i_{1}=i$, then

$$
\sum_{5 i}(z, t)=\sum_{\substack{k_{l}=0, l=1, n}}^{\infty} v_{i}\left(k+I_{j}, t\right) \prod_{l=1}^{n} z_{l}^{k_{l}} z_{i}=z_{i} \sum_{\substack{k_{l}=0, l=\overline{1, n}}}^{\infty} v_{i}\left(k+I_{j}, t\right) \prod_{l=1}^{n} z_{l}^{k_{l}}, j=\overline{1, n}, j \neq i
$$


and taking into account (16),

$$
\sum_{5 i}(z, t)=\frac{z_{i}}{z_{j}}\left[\varphi_{i}^{\Omega \backslash\{i\}}(z, t)-\left(\varphi_{i}^{\Omega \backslash\{i\}}(z, t)\right)^{\{j\}}\right] .
$$

And obviously, $\sum_{\substack{k_{l}=0, l=1, n}}^{\infty} v_{i}\left(k+I_{j}-I_{i}, t\right) \prod_{l=1}^{n} z_{l}^{k_{l}}=0$ for all $j=\overline{1, n}, j \neq i$.

Similarly, we obtain that

$$
\begin{gathered}
\left.\frac{z_{s}}{z_{c}}\left[\varphi_{c}^{\Omega \mid\{c\}}(z, t)-\left(\varphi_{c}^{\Omega \mid\{c\}}(z, t)\right)\right)^{\{c\}}\right]=0, c, s=\overline{1, n}, c, s \neq i . \\
\left.\left.\varphi_{i}^{\Omega \backslash\{i\}}(z, t)-\left(\varphi_{i}^{\Omega \mid\{i\}}(z, t)\right)\right)^{\{i\}}-\left(\varphi_{i}^{\Omega \mid\{i\}}(z, t)\right)\right)^{\{j\}}=0, j=\overline{1, n} . \\
\varphi_{c}^{\Omega \backslash\{c\}}(z, t)-\left(\varphi_{c}^{\Omega \backslash\{c\}}(z, t)\right)^{\{c\}}-\left(\varphi_{c}^{\Omega \backslash\{c\}}(z, t)\right)=0, c, s=\overline{1, n}, c, s \neq i .
\end{gathered}
$$

Consider the expression (13). Since the $j \neq i_{1} \sum_{\substack{k_{j}=0, j=1, n}}^{\infty} \prod_{l=1}^{n} z_{l}^{k_{l}}=\sum_{k_{i_{1}}=0}^{\infty} z_{i_{1}}^{k_{i_{1}}}=\frac{1}{1-z_{i_{1}}}$, in this case

$$
\begin{gathered}
R(z)=\sum_{\substack{k_{j}=0, j=1, n}}^{\infty} \alpha_{i}(k) \prod_{l=1}^{n} z_{l}^{k_{l}}= \\
=\left\{\begin{array}{l}
\left(r_{i}+\lambda_{0 i}^{+} r_{0 i}-\left(\mu_{i} p_{i 0}+\lambda_{0 i}^{-}\right) R_{i 0}+\mu_{i_{1}} p_{i_{1}}^{+} r_{i_{1} i}-\mu_{i}\left(p_{i i_{1}}^{+} r_{i i_{1}}+p_{i i_{1}}^{-} r_{i i_{1}}\right)\right) \frac{1}{1-z_{i_{1}}}, i_{1} \neq i, \\
\left(r_{i}+\lambda_{0 i}^{+} r_{0 i}-\left(\mu_{i} p_{i 0}+\lambda_{0 i}^{-}\right) R_{i 0}+\sum_{\substack{j=1 \\
j \neq i}}^{n}\left[\mu_{j} p_{j i}^{+} r_{j i}-\mu_{i}\left(p_{i j}^{+} r_{i j}+p_{i j}^{-} r_{i j}\right)\right]\right) \frac{1}{1-z_{i}}, i_{1}=i .
\end{array}\right.
\end{gathered}
$$

Taking into account (14)-(21), the system of equations (1) after the above transformations we obtain for $z$-transforms $\varphi_{i}^{\Omega \backslash\left\{i_{1}\right\}}(z, t)$ :

$$
\begin{aligned}
\frac{\partial \varphi_{i}^{\Omega \backslash\left\{i_{1}\right\}}(z, t)}{\partial t}=- & \left(\lambda_{0 i_{1}}^{+}+\lambda_{0 i_{1}}^{-}+\mu_{i_{1}}\right) \varphi_{i}^{\Omega \backslash\left\{i_{1}\right\}}(z, t)+\left(\mu_{i_{1}} p_{i_{1} 0}+\lambda_{0 i_{1}}^{-}\right) z_{i_{1}} \varphi_{i}^{\Omega \backslash\left\{i_{1}\right\}}(z, t)+ \\
& +\frac{\lambda_{0 i_{1}}^{+}}{z_{i_{1}}}\left(\varphi_{i}^{\Omega \backslash\left\{i_{1}\right\}}(z, t)-v_{i}(0,0, \ldots, 0, t)\right)+R(z)
\end{aligned}
$$


where $R(z)$ defined in equation (21). Furthermore, by solving a system of differential equations (22) and expanding the functions $\varphi_{i}^{\Omega \mid\left\{y_{1}\right\}}(z, t)$ in a power series by $z_{i_{1}}^{k_{i_{1}}}$, we can find the coefficients in these expansions $v_{i}\left(0, \ldots, 0, k_{i_{1}}, 0, \ldots, 0, t\right), i_{1}=\overline{1, n}$.

2st-Step: find $z$-transforms $\varphi_{i}^{\Omega \backslash\left\{\hat{i}_{1}, i_{2}\right\}}(z, t)$. For this recalculate all sums (3)-(10), (13), under the condition that $k_{l}=0, l \neq i_{1}, i_{2}$; expanding the found functions in a power series $z_{i_{1}}^{k_{i_{1}}} z_{i_{2}}^{k_{i_{2}}}$, find the coefficients in these expansions $v_{i}\left(0, \ldots, 0, k_{i_{1}}, 0, \ldots, 0, k_{i_{2}}, 0, \ldots, 0, t\right)$;

- continuing in this way, on $j$-th step finding $z$-transforms $\varphi_{i}^{\Omega\left\{\left\{\left\{_{1}, i_{2}, \ldots, i_{j}\right\}\right.\right.}(z, t)$, while $i_{1}, i_{2}, \ldots, i_{j}=\overline{1, n}, i_{1} \neq i_{2} \neq \ldots \neq i_{j}$; expansion coefficients $\varphi_{i}^{\Omega\left\{\left\{i_{1}, i_{2}, \ldots, i_{j}\right\}\right.}(z, t)$ in a series in powers $z_{i_{1}}^{k_{i_{1}}} z_{i_{2}}^{k_{i_{2}}} \cdot \ldots \cdot z_{i_{j}}^{k_{i_{j}}}$ give us incomes $v_{i}\left(0, \ldots, 0, k_{i_{1}}, 0, \ldots, 0, k_{i_{2}}, 0, \ldots, 0, k_{i_{j}}, 0, \ldots, 0, t\right)$; - further, continuing to make such steps, at $(n-1)$-th step find $\varphi_{i}^{\{j\}}(z, t), j=\overline{1, n}$, and at $n$-th step - $z$-transforms $\varphi_{i}(z, t)$, which satisfies (11), expanding of through a series in powers $z_{1}^{k_{1}} z_{2}^{k_{2}} \cdot \ldots \cdot z_{n}^{k_{n}}$, we can find incomes $v_{i}\left(k_{1}, k_{2}, \ldots, k_{n}, t\right)$.

\section{Example}

Consider an open HM-network with negative messages, consisting of two $n=2$ QS: $S_{1}$ and $S_{2}$. The intensity of the input stream of positive and negative messages are equals, respectively: $\lambda_{01}^{+}=0,8, \lambda_{02}^{+}=3,2, \lambda_{01}^{-}=0,6, \lambda_{02}^{-}=1,4$. The intensity of service messages at network systems are equals: $\mu_{1}=2, \mu_{2}=4$. Let the transition probabilities of messages be equals, respectively: $p_{12}^{+}=0,2, p_{21}^{+}=0,6$, and probabilities, that the positive messages served in QS $S_{i}$, sent to QS $S_{j}$, as negative messages, equals: $p_{12}^{-}=0,1, p_{21}^{-}=0,3$. The probability of escape messages from the network to the external environment be equals: $p_{10}=0,7, p_{20}=0,1$. Let the incomes from network transitions between the states be equals: $r_{1}=r_{2}=5000$, $R_{10}=7000, R_{20}=4000, r_{01}=3000, r_{02}=2500, r_{12}=10000, r_{21}=12000$.

System (1) according to the entered parameters of the network will be:

$$
\begin{gathered}
\frac{d v_{1}(k, t)}{d t}=-\left(4+2,6 u\left(k_{1}\right)+4,4 u\left(k_{2}\right)\right) v_{1}(k, t)+ \\
+1,6 v_{1}\left(k_{1}+1, k_{2}, t\right)+3,2 v_{1}\left(k_{1}, k_{2}+1, t\right)+ \\
+\left(4 u\left(k_{1}\right)+0,4\left(1-u\left(k_{2}\right)\right)\right) v_{1}\left(k_{1}-1, k_{2}, t\right)+\left(0,9\left(1-u\left(k_{1}\right)\right)+1.7 u\left(k_{2}\right)\right) v_{1}\left(k_{1}, k_{2}-1, t\right)+ \\
+1,8 u\left(k_{2}\right) v_{1}\left(k_{1}+1, k_{2}-1, t\right)+0,4 u\left(k_{1}\right) v_{1}\left(k_{1}-1, k_{2}+1, t\right)+0,2 v_{1}\left(k_{1}-1, k_{2}-1, t\right)+ \\
+21600 u\left(k_{2}\right)-16600 u\left(k_{1}\right)-1000,
\end{gathered}
$$




$$
\begin{gathered}
\frac{d v_{2}(k, t)}{d t}=-\left(4+2,6 u\left(k_{1}\right)+4,4 u\left(k_{2}\right)\right) v_{1}(k, t)+ \\
+0,8 v_{2}\left(k_{1}+1, k_{2}, t\right)+4 v_{2}\left(k_{1}, k_{2}+1, t\right)+ \\
+\left(2 u\left(k_{1}\right)+0,2\left(1-u\left(k_{2}\right)\right)\right) v_{1}\left(k_{1}-1, k_{2}, t\right)+\left(1,8\left(1-u\left(k_{1}\right)\right)+2.4 u\left(k_{2}\right)\right) v_{2}\left(k_{1}, k_{2}-1, t\right)+ \\
+0,4 u\left(k_{1}\right) v_{2}\left(k_{1}+1, k_{2}-1, t\right)+1,8 u\left(k_{2}\right) v_{1}\left(k_{1}-1, k_{2}+1, t\right)+0,9 v_{2}\left(k_{1}-1, k_{2}-1, t\right)- \\
-9800+8600 u\left(k_{1}\right)-21600 u\left(k_{2}\right) .
\end{gathered}
$$

The ratios (12) can be rewritten as follows:

$$
\begin{gathered}
\frac{\partial \varphi_{1}(z, t)}{\partial t}=11,2 \varphi_{1}(z, t)-3,6 \varphi_{1}^{\{1\}}(z, t)-7,8 \varphi_{1}^{\{2\}}(z, t)+2 z_{1} \varphi_{1}^{\{1\}}(z, t)+ \\
+\frac{1,6\left(\varphi_{1}(z, t)-\varphi_{1}^{\{1\}}(z, t)\right)}{z_{1}}+\frac{1,8 z_{2}\left(\varphi_{1}(z, t)-\varphi_{1}^{\{1\}}(z, t)\right)}{z_{1}}+\frac{3,2\left(\varphi_{1}(z, t)-\varphi_{1}^{\{2\}}(z, t)\right)}{z_{2}}+ \\
+\frac{0,4 z_{1}\left(\varphi_{1}(z, t)-\varphi_{1}^{\{2\}}(z, t)\right)}{z_{2}}+\frac{15600}{\left(z_{1}-1\right)\left(z_{2}-1\right)}-11600 \\
\frac{\partial \varphi_{2}(z, t)}{\partial t}=11,9 \varphi_{2}(z, t)-2,5 \varphi_{2}^{\{1\}}(z, t)-6,7 \varphi_{2}^{\{2\}}(z, t)+1,7 z_{2} \varphi_{2}^{\{1\}}(z, t)+ \\
+\left(2 z_{1}+1,7 z_{2}\right) \varphi_{2}(z, t)+\frac{0,8\left(\varphi_{2}(z, t)-\varphi_{2}^{\{1\}}(z, t)\right)}{z_{1}}+ \\
+\frac{1,8 z_{2}\left(\varphi_{2}(z, t)-\varphi_{2}^{\{1\}}(z, t)\right)}{z_{1}}+\frac{3,2\left(\varphi_{2}(z, t)-\varphi_{2}^{\{2\}}(z, t)\right)}{z_{2}}+ \\
+\frac{0,4 z_{1}\left(\varphi_{2}(z, t)-\varphi_{2}^{\{2\}}(z, t)\right)}{z_{2}}-\frac{28400}{\left(z_{1}-1\right)\left(z_{2}-1\right)}-3900
\end{gathered}
$$

where:

$$
\begin{gathered}
\varphi_{1}^{\{1\}}(z, t)=\varphi_{1}^{\Omega \backslash\{2\}}(z, t)=v_{1}\left(0, k_{2}\right) z_{2}^{k_{2}}, \quad \varphi_{1}^{\{2\}}(z, t)=\varphi_{1}^{\Omega \backslash\{1\}}=v_{1}\left(k_{1}, 0\right) z_{1}^{k_{1}}, \\
\varphi_{2}^{\{1\}}(z, t)=\varphi_{2}^{\Omega \mid\{2\}}=v_{2}\left(0, k_{2}\right) z_{2}^{k_{2}}, \quad \varphi_{2}^{\{2\}}(z, t)=\varphi_{2}^{\Omega \mid\{1\}}=v_{2}\left(k_{1}, 0\right) z_{1}^{k_{1}} .
\end{gathered}
$$

Make use of the algorithm described above to find the expected income of system $S_{2}$.

Determine $\varphi_{2}(z, t)=v_{2}(0,0, t)$. Find $z$-transforms $\varphi_{2}^{\Omega\left\{\left\{i_{1}\right\}\right.}$. For this we set $k_{j}=0$, $j \neq i_{1}$ and multiply the system of equations (24) to $z_{i_{1}}^{k_{i_{1}}}$ and summing over all $k_{i_{1}}$, $i_{1}=1,2$, from 0 to $\infty$. Using the sums (14)-(21), taking into account that $k_{j}=0$, $j \neq i_{1}$, from (22) we obtain relation for $z$-transforms $\varphi_{i}^{\Omega \backslash\left\{i_{1}\right\}}(z, t)$ : 


$$
\begin{gathered}
\frac{\partial \varphi_{2}^{\Omega \backslash\{1\}}(z, t)}{\partial t}=-3,4 \varphi_{2}^{\Omega \backslash\{1\}}(z, t)+2 z_{1} \varphi_{2}^{\Omega \backslash\{1\}}(z, t)+ \\
+0,8 \frac{1}{z_{1}}\left(\varphi_{2}^{\Omega \backslash\{1\}}(z, t)-v_{2}(0,0,0, t)\right)+\frac{15600}{\left(z_{1}-1\right)\left(z_{2}-1\right)}-11600, \\
\frac{\partial \varphi_{2}^{\Omega \backslash\{2\}}(z, t)}{\partial t}=-8,6 \varphi_{2}^{\Omega \backslash\{2\}}(z, t)+2,8 z_{2} \varphi_{2}^{\Omega \backslash\{2\}}(z, t)+ \\
+2,6 \frac{1}{z_{2}}\left(\varphi_{2}^{\Omega \backslash\{2\}}(z, t)-v_{2}(0,0,0, t)\right)+\frac{28400}{\left(z_{1}-1\right)\left(z_{2}-1\right)}-3900 .
\end{gathered}
$$

The solution of this system of differential equations if we specify the initial conditions $\varphi_{2}^{\Omega \backslash\{1\}}(z, 0)=\theta_{1}(z), \varphi_{2}^{\Omega \backslash\{2\}}(z, 0)=\theta_{2}(z), v_{2}(0,0,0)=V$ was obtained in Mathematica and has the form:

$$
\begin{aligned}
& \varphi_{2}^{\Omega \mid\{1\}}(z, t)=e^{\frac{t\left(0,8+z_{1}\left(-3,4 z_{1}+4,2\right)\left(z_{2}-1\right)-0,8 z_{2}\right)}{z_{1}\left(1-z_{1}-z_{2}+z_{1} z_{2}\right)}}\left\{\frac{e^{\frac{t\left(0,8-4,2 z_{1}+3,4 z_{1}^{2}\right)}{z_{1}\left(z_{1}+1\right)}}}{\left(1-z_{1}-z_{2}+z_{1} z_{2}\right)\left(0,8-4,2 z_{1}+3,4 z_{1}^{2}\right)} \times\right. \\
& \times\left[\left(z_{1}-1\right)\left(1-z_{2}\right)\left(0,8 v_{2}(0,0, t)\left(z_{1}-1\right)+0,8 V\right)\right. \\
& \left.\left.+z_{1}\left(11600\left(z_{1}\left(1-z_{2}\right)+z_{2}\right)+0,8 V+(11600-0,8 V) z_{2}\right)\right]+\theta_{1}(z)\right\}, \\
& \varphi_{2}^{\Omega \backslash\{2\}}(z, t)=e^{\frac{t\left(2,8-11,4 z_{2}+8,6 z_{2}^{2}+z_{1}\left(-2,8+11,4 z_{2}-8,6 z_{2}^{2}\right)\right)}{z_{2}\left(1-z_{1}-z_{2}+z_{1} z_{2}\right)}}\left\{\frac{e^{\frac{t\left(2,8-11,4 z_{2}+8,6 z_{2}^{2}\right)}{z_{2}\left(z_{2}+1\right)}}}{\left(1-z_{1}-z_{2}+z_{1} z_{2}\right)\left(2,8-11,4 z_{2}+8,6 z_{2}^{2}\right)^{2}} \times\right. \\
& \times\left[( z _ { 2 } - 1 ) \left(-2,8 v_{2}(0,0, t)\left(z_{1}+1\right)\left(1-z_{2}\right)+z_{2}\left(3900\left(z_{1}\left(1-z_{2}\right)+z_{2}\right)-2,8 V\right)+\right.\right. \\
& \left.\left.\left.+2,8 V-z_{1}\left(2,8 V+(3900-2,8 V) z_{2}\right)\right)\right]+\theta_{2}(z)\right\} \text {. }
\end{aligned}
$$

Initial conditions $\theta_{1}(z), \theta_{2}(z)$ satisfy the following relations

$$
\theta_{1}(z)=\varphi_{2}^{\Omega \backslash\{1\}}(z, 0)=\sum_{k_{1}=0}^{\infty} v_{2}\left(k_{1}, 0,0\right) z_{1}^{k_{1}}=\alpha z_{1}^{k_{1}^{*}},
$$

where $v_{2}\left(k_{1}, 0,0\right)=\left\{\begin{array}{c}\alpha, k_{1}=k_{1}^{*}, \\ 0, \text { in other cases, }\end{array}\right.$

$$
\theta_{2}(z)=\varphi_{2}^{\Omega \backslash\{2\}}(z, 0)=\sum_{k_{2}=0}^{\infty} v_{2}\left(0, k_{2}, 0\right) z_{1}^{k_{2}}=\beta z_{2}^{k_{2}^{*}},
$$

where $v_{2}\left(0, k_{1}, 0\right)=\left\{\begin{array}{c}\beta, \quad k_{2}=k_{2}^{*}, \\ 0, \text { in other cases }\end{array}\right.$ 
Substituting the expressions obtained for $\varphi_{2}^{\Omega_{n} \backslash\left\{i_{1}\right\}}(z, t), i_{1}=1,2$ in (26), in the Mathematica package found an analytical solution of the differential equation $\varphi_{2}(z, t)$, with the initial condition $\varphi_{2}(z, 0)=\bar{\theta}(z)$,

$$
\left.\bar{\theta}(z)=\varphi_{2}(z, 0)=\sum_{k_{1}=0 k_{2}=0}^{\infty} \sum_{2}^{\infty} v_{1}, k_{2}, 0\right) z_{1}^{k_{1}} z_{2}^{k_{2}}=\gamma z_{1}^{k_{1}} z_{2}^{k_{2}}
$$

where $v_{2}\left(k_{1}, k_{2}, 0\right)=\left\{\begin{array}{c}\gamma, k_{1}=k_{1}^{*}, k_{2}=k_{2}^{*}, \\ 0, \text { in other cases }\end{array}\right.$.

The expected income $v_{2}\left(k_{1}, k_{2}, t\right)$ of system $S_{2}$ are coefficients of expansion of the function $\varphi_{2}(z, t)$ into a double series of powers $z_{1}^{k_{1}}, z_{2}^{k_{2}}$ and it was conducted in the Mathematica package of mathematical calculations.

Let $V=0, k_{1}^{*}=k_{2}^{*}=0, \alpha=\beta=\gamma=1$, if the initial conditions are: $\theta_{1}(z)=1$, $\theta_{2}(z)=1, \bar{\theta}(z)=1$. In Figure 1 there is a graph of income $S_{2}$ at $k_{1}=2, k_{2}=2$.

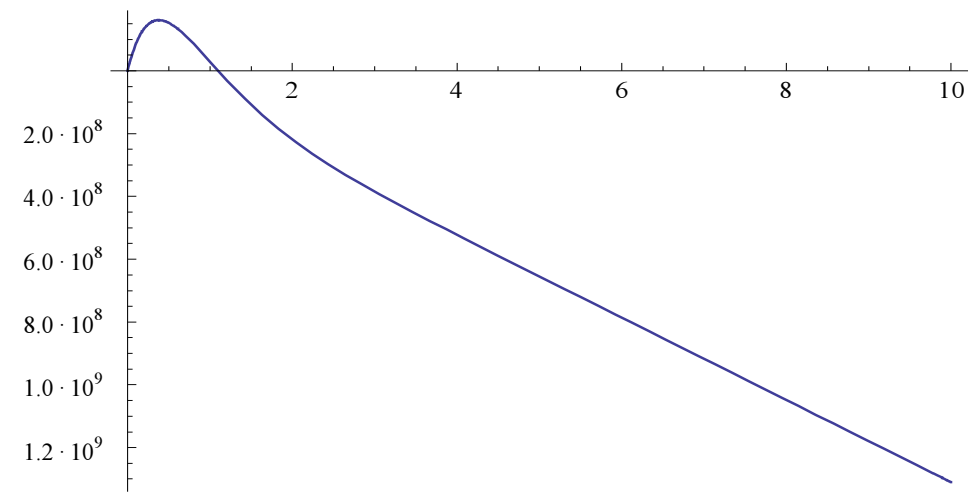

Fig. 1. Income chart $v_{2}(2,2, t)$ on interval $[0,10]$

\section{Conclusions}

The practical importance of the results lies in the fact that with the help of the proposed method one can find the expected incomes of systems considered network with an infinite number of states.

\section{References}

[1] Matalytski M., Some results of the analysis and optimization of Markov networks with incomes and their applications, Automation and Remote Control 2009, 10, 97-113 (in Russian).

[2] Gelenbe E., Product form queueing networks with negative and positive customers, Journal of Applied Probability 1991, 28, 656-663. 
[3] Bocharov P., Vishnevsky V., G-networks: the development of the theory of multiplicative networks, Automation and Remote Control 2003, 5, 46-74 (in Russian).

[4] Naumenko V., Matalytski M., Analysis networks with positive and negative messages at transition behavior. Herald of Tomsk State University. Management, Computer Science and Informatics 2013, 4, $61-70$ (in Russian).

[5] Naumenko V., Matalytski M., Analysis of Markov networks with incomes, positive and negative messages, Informatics 2014, 1, 36-44 (in Russian).

[6] Kosareva E., Matalytski M., Rozov K., Finding incomes in HM-networks with limited waiting time messages by successive approximations, combined with the method of series, Herald of Grodno State University Yanka Kupala 2012, 2, 3, 125-130 (in Russian).

[7] Naumenko V., Analysis of incomes in Markov G-networks by method of successive approximations, Herald of Grodno State UniversityYanka Kupala 2014, 2, 1 (in Russian).

[8] Matalytski M., Tikhonenko O., Koluzaeva E., Systems and QN Networks: Analysis and Application, Grodno State University, Grodno 2011, 818 p. (in Russian).

[9] Matalytski M., Koluzaeva E., Analysis of expected incomes in open network using $z$-transforms, Herald of Grodno State UniversityYanka Kupala 2008, 2, 3, 11-19.

[10] Koluzaeva E., Finding the expected incomes in the open two-node HM-network using $z$-transforms, Herald of Grodno State UniversityYanka Kupala 2010, 2, 1, 20-30. 
